\title{
Using Mathematical Models to Guide the Simulation of Improvised Explosive Devices in Public Spaces
}

\author{
Louisa Nilsen-Nygaard and Chris. W. Johnson \\ Department of Computing Science, University of Glasgow, Scotland, UK, Johnson@ dcs.gla.ac.uk
}

Keywords: Improvised Explosive Devices, Counter Terrorism.

\begin{abstract}
Terrorist attacks, for example in Madrid and London, have increased concern over the threat that Improvised Explosive Devices (IEDs) pose to public safety. Insurgent groups in Iraq and Afghanistan have developed relatively sophisticated tactics, including the use of synchronised attacks with multiple devices that have not yet been witnessed in Europe or North America. Some of these approaches specifically target the fire and rescue services. Computer simulations provide tools that can be used to plan the response to potential attacks. They can be used to work through a range of scenarios so that emergency personnel minimise their vulnerability and mitigate the threat posed to the general public. However, it can be difficult to simulate the range of human behaviours that are seen in the aftermath of terrorist attacks. Similarly, it is unclear how to develop appropriate blast and fragmentation models that capture a range of future Improvised Explosive Devices. The following pages present a brief overview of mathematical models that are being integrated into simulation tools to address these problems.
\end{abstract}

\section{Introduction}

Software simulations have been widely used to model evacuations from fire or structural collapses [1]. However, very few have been created to help analyse other hazards such as terrorist attacks. It is possible to extend some of the techniques used to create evacuation simulations to model some of the characteristics of a terrorist attack including crowd motion, blast and fragmentation patterns from explosive devices, and to help predict casualty levels in a given situation [2]. An important benefit of these tools is that they can be used in table-top exercises to work through scenarios for coordinated terrorist attacks. The threats posed by these incidents are illustrated by a suicide bombing attack on Mustansiriyah University in Iraq during January 2007. A car bomb was detonated at one of the two principle entrances to the site. This led to a partial evacuation that drew crowds to the other exit where a suicide bomber detonated their device. This is not an isolated incident. Hours before, another coordinated bombing took place in a second-hand motorcycle market in the Shia Bab al-Sheik neighbourhood of Baghdad. The first blast drew onlookers and the emergency services, who were then hit by a second explosion moments later. In these incidents, the use of evacuation procedures that were designed to protect the public from localised fires created opportunities or vulnerabilities that were exploited by the terrorists. Building occupants, spectators and the emergency services gathered at common assembly points, which were the target for secondary devices. Software simulation tools can be developed, for example, to identify ways in which evacuations can be synchronised to help disperse the crowds that otherwise create significant opportunities for terrorist attacks.

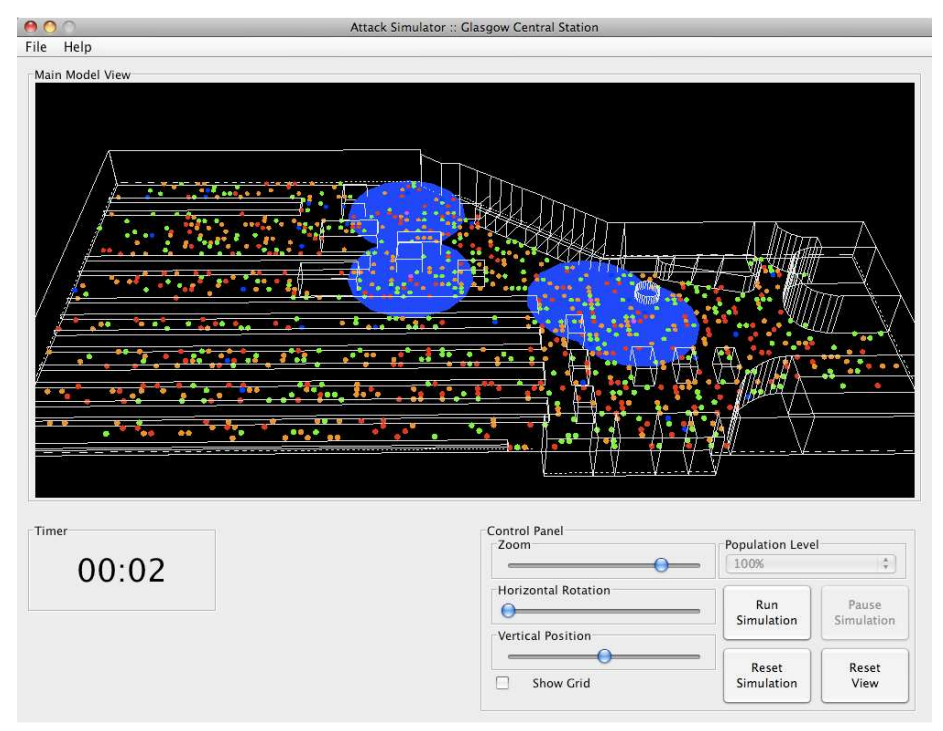

Figure 1: Interface to the Glasgow IED Simulator

Figure 1 illustrates the interface to a prototype system; the ellipses represent the extent of a potential blast as suicide bombers move inside a three dimensional model of a major UK railway station. The number of people inside these zones changes as the bombers and the other passengers move throughout the station concourse in real-time. The size of the blast and fragmentation areas can be varied to allow for larger and smaller devices given the type of explosive used. These tools can provide important information for the security services that in extremis may be forced to coordinate their response to several suspected bombers as they move through public spaces. The simulator does not account for concussive force of the blast on the people and structures in the surrounding area, although this is an obvious area for further development. However, fire and rescue services can also use the simulator to help identify scenarios in which they themselves might become the target for secondary devices as they respond to an initial incident. 


\section{High Level Models of Crowd Motion}

In order to construct simulation tools such as that illustrated in Figure 1 there must be some means of modelling complex crowd behaviours. Helbing et al [3] have used video analysis to identify patterns in crowd motion: pedestrians show an aversion to detours even when the direct route is busy; pedestrians keep a comfortable distance from other pedestrians and obstacles, but this distance will decrease if they are hurrying or the crowd is dense etc. Musse et al [4] encapsulate many of these observations in their three rules for group behaviour: 1 . Members of groups walk at the same speed; 2. Members share the same goals; 3 Members of groups will wait for each other if they are separated. These observations inform the development of different types of mathematical model that might be used to represent crowd behaviours within our software simulations.

Cellular Automata. Each person becomes an automata and individual movement is governed by a set of behavioural rules that specify permitted moves between the cells in a grid. Cellular automata models are fast and easy to implement, but automata can, typically, only move between adjacent free cells. This limits the range of crowd interactions. This approach is much more focussed on the behaviour of the individual rather than the overall emergent behaviour of groups.

Fluid dynamics. The movement of a crowd can be similar to that of a fluid; for example both a crowd and a fluid will follow the path of least resistance through a space. Henderson demonstrated the use of fluid and gas dynamics to model crowd flow in 1971 [5]. His pioneering work used the Navier-Stokes equations that describe the motion of fluids according to Newton's second law.

Particulate Models can be interpreted as a specialisation of the more general fluid dynamic models. Each agent is represented as an individual particle that navigates the environment. The individual behaviours interact to form an overall crowd behaviour. Hence an individual's movements will both shape and be shaped by the behaviours of those around them. Particular models have also modelled the environment as continuous rather than discrete space. This frees the agents from the constraints of a fixed movement lattice.

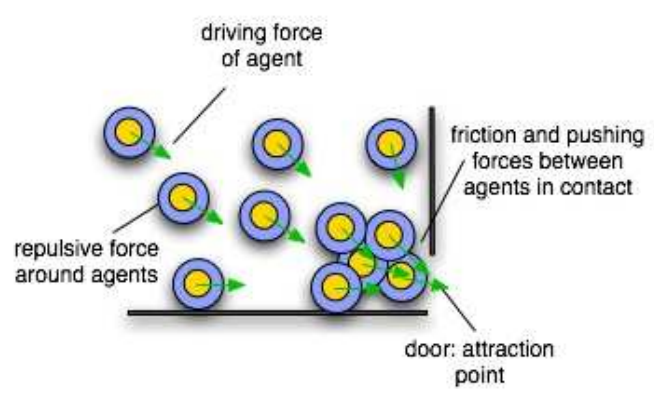

Figure 2: Illustration of social forces
A variant of particulate modelling was used to drive the simulation illustrated in Figure 1. Helbing and Molnár introduce the idea of "social forces" between individuals in a crowd. These forces include a desire to keep an acceptable distance from other. Social forces also attract them to groups as they navigate toward a shared destination [6]. This model has more recently been extended to include the physical forces that may occur in high density crowds such as pushing and friction acting upon the agents [7]. Force is essential to crowd modelling as it has a definite impact on the motion of a crowd, and carries consequences such as crush injuries in dangerous crowd scenarios. During such situations it is not uncommon to find people with severe crush injuries, and these injuries tend to occur in non-random locations such as around exits where pressure from the back of a crowd can build up to dangerous levels.

The Helbing social-physical force model does not account for the propagation of force through a crowd, nor does it allow for the consequences of force build-ups, but it does account for several forces that act upon an agent: 1. Acceleration - the velocity of an agent varies over time as it attempts to reach its optimum speed while avoiding obstacles. 2. Repulsion - there is a repulsive force between agents and between an agent and an obstacle. 3 . Attraction - agents may be attracted to other people, for example other members of their group, or by objects such as shop windows, exits etc. 4. Pushing - in dense crowds pedestrians may collide and influence each other's movement. 5. Friction - in dense crowds pedestrians can exert direct contact forces upon other pedestrians. However, it can be difficult to take these general observations of crowd behaviours and develop mathematical abstractions that can be used to drive simulation software. Let

$\begin{array}{ll}\mathrm{A} & =\text { magnitude of repulsive/attractive force, set to } 2000 \mathrm{~N} \\ \mathrm{~B} & =\text { the fall-off length of the social repulsive force, set to } \\ 0.5 \mathrm{~m} & \\ \mathrm{k} & =\text { spring constant, set to } 1.2^{*} 10^{5} \mathrm{~kg} / \mathrm{s}^{2} \\ \kappa & =\text { co-efficient of sliding friction, set to } 1 \\ \mathrm{R}_{\mathrm{ij}} & =\mathrm{r}_{\mathrm{i}}+\mathrm{r}_{\mathrm{j}}, \text { the sum of the radii of the pedestrians } \\ \mathrm{d}_{\mathrm{ij}} & =\text { the distance between their centres } \\ \mathrm{n}_{\mathrm{ij}} & =\text { the normal vector between } i \text { and } j \\ \mathrm{t}_{\mathrm{ij}} & =\text { the tangential vector to } \mathrm{n}_{\mathrm{ij}}\end{array}$

$$
\eta(x)= \begin{cases}x, & x \geq 0 \\ 0, & x<0\end{cases}
$$

The values for the constants $\mathrm{A}, \mathrm{B}, \mathrm{k}$ and $\kappa$ are taken from Lakoba et al [8]. The force equations 1-4 are described in Helbing et al [7]. One or more vectors are used to describe each force in the model. A directional vector made up of three components can describe the repulsive force between two pedestrians $i$ and $j$ : 


$$
\begin{aligned}
& \vec{f}_{i j}=\vec{f}_{\text {social repulsion }}+\vec{f}_{\text {pushing }}+\vec{f}_{\text {friction }} \\
& \vec{f}_{\text {social repulsion }}=A e^{\left(R_{i j}-d_{i j}\right) / B} \vec{n}_{i j} \\
& \vec{f}_{\text {pushing }}=k \eta\left(R_{i j}-d_{i j}\right) \vec{n}_{i j} \\
& \vec{f}_{\text {friction }}=\kappa\left|\vec{f}_{\text {pushing }}\right| \vec{t}_{i j}
\end{aligned}
$$

The form of $\eta(\mathrm{x})$ ensures that when $i$ and $j$ are not in contact the pushing and friction forces are 0 , and hence have no impact on the repulsive force. The equation for the force between a pedestrian, $i$, and a solid obstacle, $o$, is similar form to (1).

$$
\vec{f}_{i o}=A e^{\left(r_{i o}-d_{i o}\right) / B}+k \eta\left(r_{i}-d_{i o}\right) \vec{n}_{i o}+\kappa k \eta\left(r_{i}-d_{i o}\right) \vec{t}_{i o}
$$

The force of attraction to another object in the environment, including another agent or an object, can be given by reversing the social repulsion force in (1) i.e.:

$$
\vec{f}_{\text {attraction }}=-A e^{\left(R_{i j}-d_{i j}\right) / B} \vec{n}_{i j}
$$

Where $i$ is the individual under consideration and $j$ is the attracting person or object. The force of acceleration in the simulation is modelled by an equation for personal velocity, which changes over time. It also takes into account the velocity of the crowd in the immediate vicinity of the individual, the "collective velocity", which can impact on the preferred velocity e.g. a slow moving crowd will slow down a faster individual. The ability to avoid some objects and also to model the attractive forces of other individuals is particularly important for an IED simulator where we would like to model the intervention of counter terrorism agencies and also of the fire and rescue services. The opening sections have described recent coordinated suicide attacks can target the crowds that gather in assembly points after an initial evacuation. These potential risks can be mitigated by the marshalling strategies after suspected terrorist attacks. The impact of these techniques can be modelled using attraction and repulsion forces.

$$
\begin{aligned}
& \vec{f}_{\text {preferred }}=-m \frac{\vec{v}-\overrightarrow{v_{0}}}{\tau_{i}} \\
& \vec{v}_{0}=(1-p) v_{0} \vec{e}_{i}+p\left\langle\overrightarrow{v_{j}}\right\rangle_{i}
\end{aligned}
$$

Where

$$
\begin{array}{ll}
\underset{\mathrm{m}}{\vec{v}} & =\text { mass of } i \\
\vec{v} & =\text { current velocity } \\
& =\text { reaction time }
\end{array}
$$

$\overrightarrow{v_{0}} \quad=$ the preferred velocity

$v_{0} \quad=$ the desired isolated velocity of $i$

$\vec{e}_{i} \quad=$ the unit vector in $i$ 's direction of motion

$\left\langle\overrightarrow{v_{j}}\right\rangle_{i}=$ average velocity $i$ perceives in a $2-3 \mathrm{~m}$ radius

$p \quad=$ determines weights of own and collective velocities

In the IED simulator the 'driving' force that acts on each person is derived from the attractive force that acts to move them towards their current goal. This could be another agent, such as a first responder, or an exit or any point on a preferred route through their environment.

\section{Movement, Collisions and Group Behaviour}

The particle motion approach to crowd modelling embedded within the IED simulator can be broken down into three broad tasks - movement, collision avoidance, and group behaviours.

Movement forces direct the progress of an agent through their environment. Each individual is assumed to have a starting point and their motion is mainly driven by an attraction to a destination. There may also be obstacles to avoid, see below. Perfect knowledge of the environment is not assumed, therefore the complete route is not calculated. An agent moves towards their destination using a directed search algorithm, this can be depth first, breadth first or hybrid techniques depending on the implementation. Some way-points have actions associated with them, for example if a shop is a goal then the agent may incur a waiting time to simulate shopping before being assigned a new goal. This is again important for counter-terrorism models where people are not simply walking between points in the environment when a device is detonated. Previous attacks have been focussed on shopping areas, markets and on political rallies where significant portions of a crowd are not moving at the moment of initial detonation [2].

Collision Avoidance. As individuals navigate through their environment they will have to avoid collisions with other agents and static objects. Collision avoidance is achieved by monitoring obstacles within an 'area of influence', followed by an alteration of the agent's trajectory to avoid them. The area of influence needs to be properly sized to allow for detection to occur while there is still enough time to alter the agent's path, and is therefore contingent on the velocity of the agent. This aspect of the model is essential in the moments after an IED attack given that individuals and groups may have to navigate around an environment that is very different from the one immediately before an explosion. They may also be forced to move around emergency personnel as they provide assistance in the aftermath of an attack. The avoidance of static objects is relatively simple. The trajectory of an individual $i$ can be altered by the application of an avoidance force [9] to miss obstacle $o$ : 


$$
\vec{f}_{\text {avoidance }}=\frac{\left(d_{i k} \times v_{i}\right) \times d_{i k}}{\left|\left(d_{i k} \times v_{i}\right) \times d_{i k}\right|}
$$

Avoiding collisions with other pedestrians is more complicated. The simplistic approach could be more correctly termed 'collision detection'; when two agents are about to collide one will simply stop moving, generally the slower or less aggressive one, and allow the other to pass. This approach does not produce an adequate simulation of crowd motion. The second approach is similar to that of the static obstacle avoidance and involves altering the trajectory of one agent to avoid the other entirely. The 'area of influence' mentioned above is used to detect approaching agents and once again an avoidance force is calculated to alter the trajectory if there is sufficient time to do so. Otherwise the simplistic method is used and one agent must stop in order to avoid the collision. Several parameters affect the avoidance forces on an individual. These include the distance to obstacles, the direction vector of the colliding agent relative to the current direction vector of the avoiding agent, and the density of the crowd in the area. The rational of the equation used to obtain the avoidance force is described in detail in Pelechano et al [9] but the tangential force that will steer pedestrian $i$ to avoid pedestrian $j$ is given by:

$$
t_{j}=\frac{\left(d_{i j} \times v_{i}\right) \times d_{i j}}{\left|\left(d_{i j} \times v_{i}\right) \times d_{i j}\right|}
$$

This tangential force is then multiplied by two scalar weights to obtain the agent avoidance force that will alter the trajectory:

$$
\begin{aligned}
& \vec{f}_{i j}=t_{j} w_{i}^{d} w_{i}^{o} \\
& w_{i}^{d}=\left(d_{i j}-D_{i}\right)^{2} \\
& w_{i}^{o}= \begin{cases}1.2, & \left(v_{i} \cdot v_{j}\right)>0 ; \\
2.4 & \text { otherwise }\end{cases}
\end{aligned}
$$

$w_{i}^{d}$ is due to the distance between the agents, and will increase in weight as the agents get closer because the trajectory will have to be more radically altered to avoid collision. $w_{i}^{o}$ is due to the differences in orientation of the agents' velocity vectors (the driving force), the weight is greater if the colliding agent is travelling in the opposite direction. $D_{i}$ is the detection distance of the pedestrian and controls the size of the detection area. This allows the density of the crowd to be factored in; the distance at which collisions can be detected decreases as density increases, therefore the value of $D_{i}$ should decrease as density increases.

Group Behaviours Individuals within a crowd often seem to be influenced by the movements of groups around them [3, 4]. For the purposes of our prototype IED simulator a group consists of between 2 and 8 people [12]. Each group has a randomly allocated 'leader' that, all other things being equal, will be followed by the other members of the group via an attractive force, and a group identifier. Current implementations do not model the formation of predetermined groups e.g. friends who run into each other. This might be appropriate in the aftermath of a blast in a nightclub district, such as the area affected by the Bali blasts, where individuals struggle to find their friends. This is, arguably, less relevant in an attack on a railway station where most groups of travellers will be close together immediately before any incident.

Individuals and groups can be influenced to follow a path that has already been traversed by others [3]. This is distinct from 'flocking' because individuals will also follow some time after a group or person has already taken a particular route. This type of behaviour results in the formation of lanes of pedestrians through a crowd. These lanes are often the only way that people can get through a congested area such as a narrow corridor or dense crowd. Again, this is important in the context of counter terrorism given that such congested areas may themselves be the targets of secondary devices. 'Following' behaviour can be modelled using residual direction fields; as an individual moves through an area, an imprint of their direction is left which exerts a small force on the direction of the next pedestrian to move through the area. The imprint fades over a few seconds if not replaced by that of another pedestrian, but the effect can be cumulative over time to have a greater influence over agents to follow the path. 'Following' behaviours can combine with obstructions to create queues. These form at popular points where bottlenecks form and individuals are forced to slow down. Fans are created when multiple queues are created by different 'lanes' towards a common obstruction or 'pinch point'. A key concern for the end-users of our system is to train first responders to minimise the delays created by the formation of these group structures. Simulations can help senior personnel to gain an overview of the interactions between their staff and these different crowd behaviours that present targets for subsequent terrorist attacks.

\section{Blast and Fragmentation}

For any explosive device it is possible to calculate the radius of the blast, the effective range of fragmentation, and the distance from the device at which specific "overpressures" will occur. The pressure in excess of the normal atmospheric pressure, called overpressure, is used to measure blast effect in pounds per square inch (PSI). The effects of ranges of overpressures on people and buildings have been observed, so the damage at a certain range can be predicted with relative accuracy, although damage to structures is highly contingent on materials and construction. The explosive force of a device can be calculated using the TNT (Trinitrotoluene) equivalence method, which draws on a large body of experimental data to predict the consequences of explosions resulting from other volatile materials by calculating the equivalent mass of TNT. The consequences can then be found from various tables of empirical data. The PEAC-WMD 
2007 tool from AristaTek Inc (http://www.aristatek.com/) uses this method to calculate the distance at which specific overpressures will occur for a given mass of explosive material. Casualties resulting from blast alone are unlikely to occur below an overpressure of 4.5 PSI. In terms of casualty projections for the simulated environment the range of 4-25 PSI is the most important. At 25 PSI, total destruction of buildings can be anticipated with $99 \%$ human fatality. Most of our modelling work has focused on devices that are worn as a belt or vest. These garments are packed with cylinders or plates of explosive surrounded by metal fragments, such as ball bearings, nails or screws. Shrapnel is responsible for around $90 \%$ of the casualties from these devices.

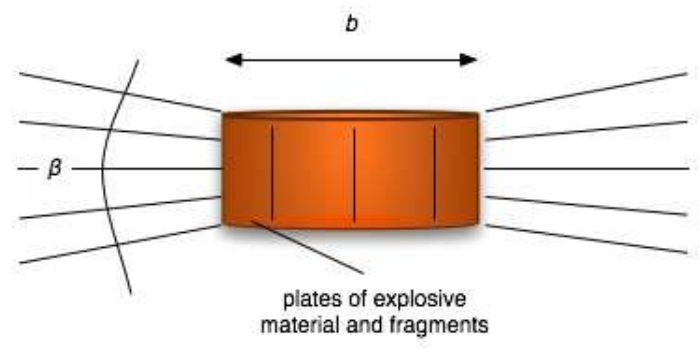

Figure 3: Construction of a typical suicide belt, showing the angle of fragment dispersion

Industrial explosives such as C4 and TNT are popular because they are easy to handle. However, there are risks for terrorists in obtaining the explosives before an attack. Other 'homebrew' substances such as TATP (Triacetone Triperoxide) are relatively easy to make from commonly available ingredients. However, TATP is sensitive to heat, friction and shock. Ammonal is a more stable homemade compound derived from ammonia nitrate (fertilizer) mixed with coal and aluminium dust. This is commonly used as the bulk component detonated with a small amount of TATP. The simulation in Figure 1 models attacks using this type of hybrid TATP-Ammonal device. We assume a suicide belt may hold around $4.5 \mathrm{~kg}$ of explosives, a vest $9 \mathrm{~kg}$, and a briefcase around $23 \mathrm{~kg}$. These default values can, however, be varied for each run of the simulator. The PEAC-WMD software has been used to produce figures for the range of specific overpressures from the device in order to ascertain the damage that would occur at certain distances.

For the likely mass of explosive contained in a suicide device, most blast casualties will be within a 10 metre radius, assuming there are no protective structures between them and the explosive [10]. The railway station illustrated in Figure 1 is, however, full of permanent and semi-permanent structures that could offer some degree of protection to individuals in the model. These include concession booths, benches, large signs etc. Following the PEAC-WMD model, we assume that there is a $1 \%$ probability of fatality for individuals within an overpressure area of 4.5 PSI, and a $99 \%$ fatality rate at 25 PSI. The expected casualty rate between these values is difficult to determine. Kress has recently developed mathematical models that can be used to refine these approximations by developing probability distributions for the number of casualties based on the density of the crowd around the suicide bomber at the time of detonation [11]. This approach considers the impact of shrapnel, which can be determined by the number of fragments packed into the device, the distance over which it is effective, the dispersion angle of the fragments, the density of the crowd surrounding the bomber etc.

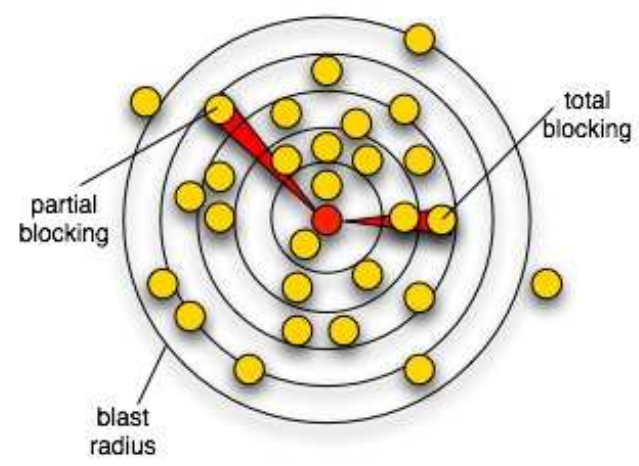

Figure 4: Arena of a suicide bomb

Our implementation of the Kress model is based on the concept of an arena, which describes the effective range of the fragments produced by an IED. The radius of the arena, $\mathrm{R}_{0}$, is the same size as the fragmentation range [10]. This radius is larger than the effective range of the blast, supporting the assertion that the fragmentation is more dangerous than the blast itself. The arena is divided into a sequence of $\mathrm{M}$ concentric circles, each the width of $b=a$ person's diameter, giving $M=R_{0} / b$. The innovative feature of our implementation is that we can continuously update the Kress values in real-time as the simulated crowd moves within a potential target area. When people walk between different areas of the railway station concourse, the number of potential casualties will change as the crowd densities change. The user of the simulation can assess the impact of potential group behaviours and different occupancy levels on the casualties from a coordinated terrorist attack. Let

$$
\begin{aligned}
& \mu_{\mathrm{m}} \quad=\text { number of people in a ring, } \\
& \text { observable from simulation } \\
& \alpha(\mathrm{m})=\text { probability that an exposed } \\
& \text { target in ring } m \text { is exposed } \\
& \mathrm{N} \quad=\text { number of effective fragments } \\
& \mathrm{L} \quad=\text { number of people in the arena } \\
& \mathrm{A}=\text { the exposed area of a person } \\
& \mathrm{K}(\mathrm{M})=\text { the maximum number of people } \\
& \text { possible in the arena } \\
& \mathrm{P}_{\mathrm{H}}(\mathrm{m})=\text { probability that exposed } \\
& \text { person in ring } m \text { will be hit } \\
& \beta=\text { dispersion angle of fragments }
\end{aligned}
$$


Assuming total crowd blocking, meaning that anyone whose position is blocked will not be injured, $\mathrm{E}_{\mathrm{m}}$ casualties can be expected in the $m^{\text {th }}$ ring of the arena, with $\mathrm{E}_{\mathrm{m}}$ given by:

$$
E_{m}=\mu_{m} \times \alpha(m) \times P_{H}(m)
$$

Therefore the total number of casualties $\mathrm{E}(\mathrm{M})$ due to fragmentation can be assumed to be:

$$
E(M)=\sum_{m=1}^{M} E_{m}
$$

The three terms of equation 1 can be calculated from information available from the simulation and from data provided by the user. The first term $\mu_{\mathrm{m}}$ is the number of people in the $m^{\text {th }}$ ring, which is observable. The second term $\alpha(\mathrm{m})$, is calculated by:

$$
\alpha(m)=\left\{\begin{array}{l}
\prod_{l=1}^{L-1}\left(1-\frac{m-1}{K(M)-l}\right), L<K(M)-m+2 \\
0
\end{array}\right.
$$

Where $\mathrm{K}(\mathrm{M})$ is given by:

$$
K(M)=\sum_{m=1}^{M} \frac{\pi}{\arcsin \left(\frac{1}{2 m}\right)}
$$

The Kress model does not address blast related injuries. Our implementation, therefore, combine the expected casualties from his model with the overpressure calculations summarised in the previous section to derive overall casualty projections. Further work is required to determine the best means of combining these two approximations. Summing the casualties produced from blast and fragmentation would produce an over-estimate given that some victims may be injured by both over-pressure and by projectiles produced in the aftermath of any detonation.

\section{Conclusion}

The work described in this paper represents initial steps towards the development of counter-terrorism simulation tools. It is particularly important to validate predicted injury patterns and crowd behaviours from more complex, mathematical models. However, it can be difficult to gather detailed information about fragmentation and blast patterns in the aftermath of terrorist attacks when the priority is to protect the public and tend to casualties Even at this early stage in our work, however, it is apparent that the patterns of attack are changing rapidly in response to recent changes in strategy by emergency services and military organisations. Children and the disabled are being used in the synchronised deployment of IEDs. Multiple devices are being used to support political assassinations of as individual targets move through crowds of supporters. Counter terrorism agencies must take considerable pains to guard against these forms of attack without imposing unnecessary restrictions on civil liberties. Unless we develop tools that reflect the flexibility and ingenuity of those who deploy these devices then there is little prospect that we will be able to protect the public from future terrorist attacks.

Acknowledgement: This work was produced as part of an EPSRC/BAe Systems CASE studentship.

\section{References}

[1] E.R. Galea, Proposed Methodology for the Use of Computer Simulation to Enhance Aircraft Evacuation Certification. AIAA Journal of Aircraft, Vol 43, Number 5, pp 1405-1413, 2006.

[2] C.W. Johnson and L. Nilsen-Nygaard, Extending the Use of Evacuation Simulators to Support Counter Terrorism. To appear in the International Systems Safety Conference, Vancouver, Canada, 2008.

[3] D. Helbing, P. Molnár, I. Farkas, K. Bolay, Self Organizing Pedestrian Movement, Environment and Planning B: Planning and Design, 28:361-383, 2001

[4] S.Musse, C. Babski, T. Capin, D. Thalman, Crowd Modelling in Collaborative Virtual Environments, Proc. ACM Symp. Virtual Reality Software and Technology, 115-123, 1998

[5] L. Henderson, The Statistics of Crowd Fluids, Nature, 229:381-383, 1971

[6] D. Helbing, P. Molnár, Social Force Model for Pedestrian Dynamics, Physical Review, 51:4282-4286, 1998

[7] D. Helbing, I. Farkas, T. Vicsek, Simulating Dynamical Features of Escape Panic, Nature, 407:487-490, 2000

[8] T.I. Lakoba, D.J. Kaup, N.M. Finkelstein, Modifications of the Helbing-Molnár-Farkas-Vicsek Social Force Model for Pedestrian Evolution, Simulation Vol. 81, No. 5, 339-352, 2005.

[9] N. Pelechano, J.M.Allbeck, N.I.Badler, Controlling Individual Agents in High-Density Crowd Simulation, ACM SIGGRAPH Symposium on Computer Animation, 2007

[10] PEAC-WMD 2007, AristaTek. http://www.aristatek.com, last accessed March 2008 .

[11] M. Kress, The Effect of Crowd Density on the Expected Number of Casualties in a Suicide Attack, Naval Research Logistic, Vol. 52, No. 1, 22-29, 2005.

[12] R. Sommer, Personal Space, Englewood Cliffs, Prentice Hall, 1979 